\title{
Easy resolution of severe obstructive kidney injury
}

1. Internal Medicine Service, Centro Hospitalar Tondela-Viseu, Viseu, Portugal 2. Internal Medicine Service, Hospital de Braga, Braga, Portugal

http://dx.doi.org/10.1590/1806-9282.65.3.333

\section{SUMMARY}

Chronic constipation is a common diagnosis with a high prevalence in the elderly. Constipation affects the quality of life of sick individuals, bringing several clinical complications.

Keywords: Constipation. Elderly. Acute Kidney Injury.

\section{CASE}

We present the case of an 82-year-old caucasian woman living in a nursing home who went to the ER due to strong sputum and fever. She had a previous history of controlled high blood pressure, early-stage dementia, and chronic constipation due to laxatives. She presented leukocytosis and severe kidney injury with a creatinine of $3.4 \mathrm{mg} / \mathrm{dL}$ and urea of $231 \mathrm{mg} / \mathrm{dL}$. To clarify the situation, a computed abdominal and pelvis axial tomography was made, which revealed a prominence of the excretory channels on the left side of both kidneys (figure 1) and prominent ureters in the proximal segment conditioned by the dilation of the sigmoid and colon with extensive coprostasis and exuberant fecaliths (figure 2). After eliminating the fecaliths with cleaning enemas, the patient presented normalized kidney function with a creatinine of $1.1 \mathrm{mg} / \mathrm{dL}$ and urea of $60 \mathrm{mg} / \mathrm{dL}$.

\section{DISCUSSION}

Chronic constipation is a common diagnosis in the elderly with a prevalence of $30 \%$ to $40 \%$ in patients over 65 years old; it is also more common among women. For patients living in nursing homes, the prevalence increases to $70 \%$. Constipation affects the quality of life of sick individuals, causing many clinical complications and increasing healthcare expenses. ${ }^{1,2}$ In Portugal, for instance, over 10 million 


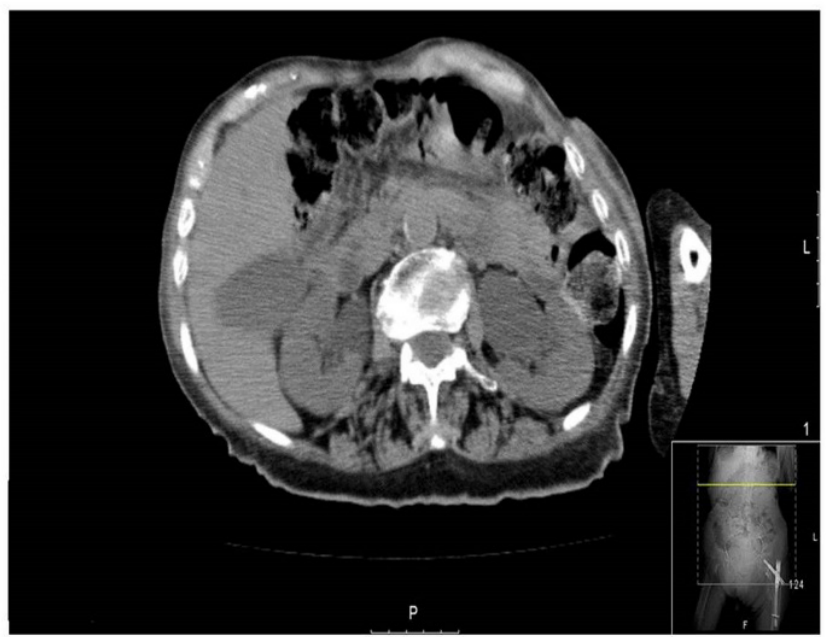

FIGURE 1: PROMINENCE OF THE EXCRETORY CHANNELS OF BOTH KIDNEYS AND PROMINENT URETERS.

euros are spent annually on laxatives and more than 50,000 medical appointments. ${ }^{3}$ In the USA, 820 million dollars are spent on laxatives, and in the UK, over 48 million pounds, thirty times the amount spent on high blood pressure treatment. ${ }^{2}$

Constipation is associated with complications such as symptoms of the inferior urinary tract and fecal impaction, which may lead to perforation of the colon. The complications are more frequent in hospitalized or bedridden patients with neurodegenerative diseases. ${ }^{1,2}$

One of the aspects of constipation that makes it difficult to deal with is the absence of an agreed-upon definition. The most common criterion is the limited number of three evacuations a week, although the main complaints are due to hard or irregular feces and an excessive effort in defecation. ${ }^{1,2}$ The Rome III criteria are currently the most commonly used. ${ }^{2,4}$

It is important to clearly make a distinction between primary constipation - which may be divided into normal intestinal transit, slow intestinal transit, and dysfunction of the pelvic pavement - and secondary constipation due to medicines, neuropathic and myopathic alterations, hydro electrolyte

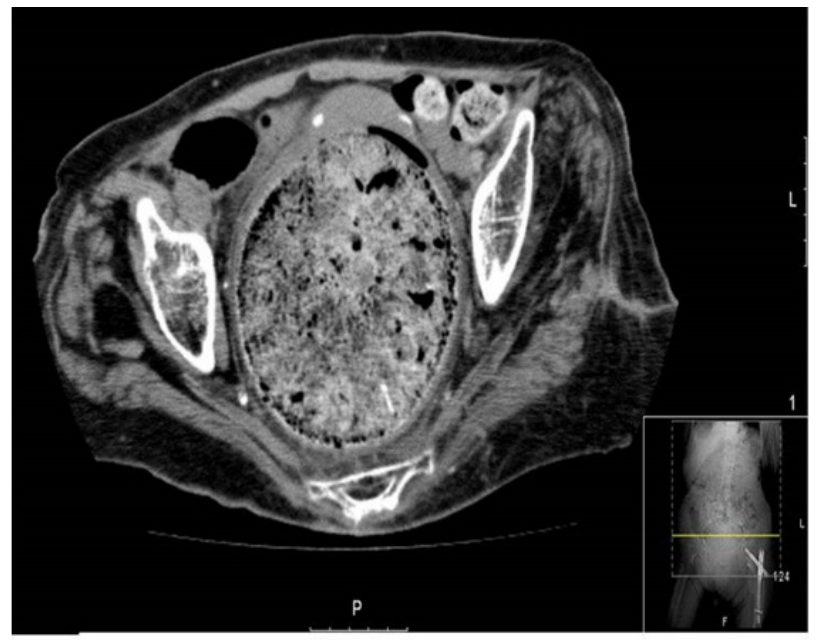

FIGURE 2: EXUBERANT FECALITHS

alterations, and intestinal and endocrine-metabolic diseases. The therapeutic strategy varies according to the etiology. ${ }^{1,2}$

Currently, there are no guidelines for treating constipation. Nonpharmacological measures depend on a lifestyle change with increased ingestion of liquids and fiber, as well as physical exercise. However, these remedies are often ineffective in the elderly, so they require a multifactorial approach. The pharmacological measures include the use of laxatives, which should be prescribed individually bearing in mind the elderly's cardiovascular and renal conditions and their regular medication. In cases of constipation resistant to common laxatives, new agents such as lubiprostone, linaclotide, plecanatide, prucalopride, and elobixibat are available. These new agents have proven to be safe and efficient in adults but are only partially validated for the elderly. ${ }^{1,2}$

We insist on the importance of creating guidelines that define constipation and its treatment in the elderly so these patients' quality of life can be genuinely improved and complications avoided.

All the authors contributed to the manuscript and read the final version.

There is no conflits of interest.

\section{RESUMO}

A obstipação crônica é um diagnóstico com alta prevalência comum em idosos. A constipação afeta a qualidade de vida das pessoas doentes, carregando muitas complicações clínicas. 


\section{REFERENCES}

1. De Giorgio R, Ruggeri E, Stanghellini V, Eusebi LH, Bazzoli F, Chiarion $G$. Chronic constipation in the elderly: a primer for the gastroenterologist. BMC Gastroenterol. 2015;15:130.

2. Couto JRS. Obstipação crónica no idoso: opções terapêuticas [Dissertação de mestrado]. Coimbra: Faculdade de Medicina da Universidade de Coimbra; 2014. 79p.
3. Villanueva T, Alves MM. Obstipação intestinal e impactação fecal. Programa Harvard Medical School Portugal. [Cited 2018, April 28]. Available from: https://hmsportugal.wordpress.com/2012/04/10/obstipacao-intestinal-e-impactacao-fecal/

4. Bharucha AE, Pemberton JH, Locke GR $3^{\text {rd }}$. American Gastroenterological Association technical review on constipation. Gastroenterology. 2013;144(1):218-38 